\title{
Procedimiento para el cálculo de los parámetros de un modelo térmico simplificado del motor asincrónico
}

\author{
Parameter estimation procedure for an asynchronous \\ motor simplified thermal model
}

\author{
Julio R. Gómez Sarduy ${ }^{1}$ \\ Marcos A. de Armas Teyra ${ }^{1}$ \\ Percy R. Viego Felipe ${ }^{1}$ \\ Michel García Abreu ${ }^{2}$ \\ Recibido 24 de febrero de 2010, aceptado 7 de marzo de 2011 \\ Received: February 24, $2010 \quad$ Accepted: March 7, 2011
}

\begin{abstract}
RESUMEN
En este trabajo se presenta un método para estimar las conductancias y capacitancias de un modelo térmico simplificado del motor asincrónico, utilizando una técnica de baja invasividad. El procedimiento permite predecir el incremento de temperatura del estator del motor asincrónico, tanto para régimen dinámico como en condiciones de estabilidad térmica. Se basa en la estimación paramétrica mediante un modelo de referencia, utilizando como optimizador un algoritmo genético (AG). Se logra en definitiva obtener los parámetros del modelo térmico con un ensayo más sencillo que lo requerido por otros métodos experimentales complejos o cálculos analíticos basados en datos de diseño. El procedimiento propuesto se puede llevar a cabo en condiciones propias de la industria y resulta atractivo su empleo en el análisis de calentamiento de estas máquinas. El método se valida a partir de un estudio de caso reportado en la literatura y se aplica a un caso real en la industria, lográndose una buena precisión.
\end{abstract}

Palabras clave: Modelo térmico, motor asincrónico, temperatura, estimación de parámetros, algoritmo genético.

\section{ABSTRACT}

In this paper, an asynchronous motor simplified thermal model method for conductances and capacitances estimation is presented. A low invasive technique is used. The developed procedure allows the stator temperature rise prediction, not only for dynamic regimes, but also in case of thermal stability. A parametric estimation is done through a reference model, using a genetic algorithm (GA) as optimizing method. The thermal model parameters are finally obtained with an easer experimental work, than the required by other complex experimental methods or by analytical calculations based on design data. The proposed procedure can be carry out in the particular conditions of industrial environment. Its application is specially useful for asynchronous machine thermal analysis. Using the data of a study case reported in literature, the method validation is done, and is applied in an industrial real case, with good precision resulted from it.

Keywords: Thermal model, asynchronous motor, temperature, parameter estimation, genetic algorithm.

\footnotetext{
1 Centro de Estudios de Energía y Medio Ambiente. Universidad de Cienfuegos. Carretera a Rodas. Cienfuegos, Cuba. E-mail: jgomez@ucf.edu.cu; pviego@ucf.edu.cu; marmas@ucf.edu.cu

2 Departamento de Física-Química. Universidad de Cienfuegos. Carretera a Rodas. Cienfuegos, Cuba.

E-mail: mgabreu@ucf.edu.cu
} 


\section{INTRODUCCIÓN}

Los estudios de calentamiento de motores eléctricos se enfocan en dos direcciones: al diseño o a su operación. Estos análisis térmicos están ligados al concepto de eficiencia. Por otro lado, se han incrementado los requerimientos para que los fabricantes desarrollen motores más pequeños y eficientes; además, una vez que el motor se encuentra en operación, su vida útil depende en buena medida del sobrecalentamiento del aislamiento. Debe también señalarse que se requieren análisis térmicos para determinar con mayor exactitud la reducción de potencia necesaria cuando el motor está sometido a condiciones de calidad del suministro eléctrico diferentes a las especificadas por las normas internacionales vigentes [1-3].

Algunos análisis térmicos precisos se han obtenido utilizando métodos de elementos finitos y proveen un mapa de temperatura detallado e información sobre el comportamiento térmico en estado transitorio. Sin embargo, estos modelos son mayoritariamente útiles para el diseño, son procedimientos costosos desde el punto de vista computacional y pueden ser solamente utilizados para modelar la transferencia de calor por conducción en elementos sólidos [4]. Por esta razón, se emplean con frecuencia modelos de parámetros concentrados, con diferentes simplificaciones, según el caso que se desea tratar. Las técnicas utilizadas para determinar los parámetros de estos modelos van desde el empleo de los datos de catálogo [5], hasta ensayos complicados para condiciones de campo o combinaciones de ensayos con información de diseño de los elementos del motor [1, 3, 5-7]. En función de los objetivos buscados, se ha desarrollado una gran cantidad de modelos térmicos, cada uno de los cuales exige distintos tipos de parámetros y ensayos para su determinación.

En [6] por ejemplo, se presenta un modelo térmico de parámetros concentrados que solamente utiliza las temperaturas del estator y del rotor para incluirlas como corrección en la estimación paramétrica on-line de un motor para aplicaciones de control vectorial sin sensores. Para identificar sus parámetros se llevan a cabo ensayos con corriente directa para determinar las conductancias térmicas; y ensayos con corriente alterna para determinar las capacitancias y los coeficientes de variación de las conductancias con la velocidad. Este modelo es incluido en un estimador paramétrico on-line, de un clásico control vectorial indirecto, para mejorar la precisión del mismo en estado estable.

En [7] el modelo simplificado que se presenta es utilizado posteriormente para calcular la reducción de la potencia ("derating") cuando se alimenta el motor desde un variador de velocidad PWM. La mayoría de los parámetros del circuito térmico son calculados a partir de expresiones analíticas que requieren las dimensiones principales del motor (radios interior y exterior de la culata del estator, radio del mandrilado del estator, radios interior y exterior del núcleo del rotor, la longitud axial del núcleo), así como los coeficientes de conductividad térmica de diferentes materiales. O sea, requiere información relacionada con el diseño del motor y los materiales empleados que no siempre se encuentra disponible. Por otro lado, algunos parámetros requieren ser ajustados posteriormente mediante ensayos con tensión directa. Este modelo es solo para estado estable y permite predecir el sobrecalentamiento con discrepancias de $\pm 5^{\circ} \mathrm{C}$.

En [8] se describe un modelo sencillo que se reduce a dos ecuaciones diferenciales para estimar la temperatura del estator y del rotor, pero se plantea la necesidad de seis ensayos: dos alimentando el motor con una fuente de corriente directa a diferentes tensiones; dos con corriente alterna a distintas frecuencias y con el rotor bloqueado; y otras dos con corriente alterna a diferentes frecuencias pero con cierta carga. En [9] se proponen tres ensayos que permiten obtener un circuito térmico equivalente generalizado del motor asincrónico. El primer ensayo es simplemente una prueba en vacío. El segundo ensayo requiere la operación del motor en un régimen especial, en el que es accionado por un motor de corriente directa mientras se alimenta con tensiones de secuencia de fase inversa, de manera que el campo que se produce gire contrario a la rotación del motor. El tercer ensayo requiere la conexión en serie de las fases del estator, mientras se alimenta el motor con una fuente de corriente directa. Aunque se reporta en esa investigación una precisión dentro de $2-3^{\circ} \mathrm{C}$ en la estimación, el modelo no es dinámico y solo es útil para predecir el incremento de temperatura de estado estable y a plena carga.

En [1] se muestra un modelo que permite el análisis térmico del motor en régimen desbalanceado con el 
objetivo de estimar la pérdida de vida útil, pero el método de determinación de parámetros requiere tres ensayos, dos para determinar las conductancias y uno para las capacitancias. El primer ensayo consiste en alimentar una fase con tensión de corriente directa a corriente nominal, dejar que se estabilice la temperatura y medirla para cada fase por el método de la resistencia. El segundo ensayo necesita conectar los devanados en serie y alimentarlos con tensión directa a corriente nominal; y el tercer ensayo es similar al primero pero tomando los valores de resistencia a intervalos, hasta que la temperatura alcanza el valor final.

Como puede apreciarse, estos métodos requieren ensayos de laboratorio con equipamiento especial y son altamente invasivos. En este trabajo, se propone un método para estimar los parámetros de un modelo térmico simplificado que permite predecir incrementos de temperatura del estator con menor invasividad que los métodos reportados hasta el momento.

\section{ANÁLISIS TEÓRICO}

\section{Modelo térmico del motor asincrónico}

En este estudio para calcular el incremento de temperatura del motor a un estado de carga determinado se adopta un modelo térmico que se deriva del empleado en [1]. En el modelo planteado se desacoplan los circuitos térmicos del rotor y del estator. Es bueno aclarar, que aunque la transferencia de calor a través del entrehierro puede ser tratada más rigurosamente, bajo ciertas circunstancias es despreciada; por ejemplo, cuando por cálculo se demuestra que es muy pequeña o en motores en cuyo diseño se incluyen canales de ventilación radiales [10]. En otros casos, la temperatura dentro de la máquina se considera uniforme y también se tratan los circuitos térmicos por separado [11]. En este trabajo se supone como en [1], que el calor del rotor fluye al ambiente a través del eje del motor y no hacia el estator, o sea, que el entrehierro constituye una barrera a la transferencia de calor entre el estator y el rotor y viceversa [12]. Debido a que la vida útil del motor depende de la vida del aislamiento del estator, el enfoque se centra en este último y no se determinan los parámetros del circuito térmico del rotor. No obstante las simplificaciones realizadas, como se verá posteriormente y también se verifica en [1], no se afectan los resultados del modelo.
Ha sido extensamente utilizada la práctica de representar los complejos parámetros térmicos distribuidos de los motores eléctricos mediante circuitos de parámetros concentrados. En las Figuras 1 y 2 se reproducen estos circuitos para el estator y el rotor respectivamente, en los cuales se representan las diferentes fuentes de calor, capacitancias y conductancias térmicas. El significado de los términos empleados en estas figuras y sus unidades se explican en la Tabla 1.

Los modelos térmicos del estator y del rotor son similares y están constituidos por dos fuentes de calor y dos conductancias. En ambos circuitos, las fuentes de calor son debidas a las pérdidas en el devanado y el núcleo y las conductancias se refieren

Tabla 1. Significado de los términos empleados en el modelo térmico del motor.

\begin{tabular}{|c|c|}
\hline Nombre & Descripción \\
\hline$P c u$ & Pérdidas de cobre del estator, en W. \\
\hline$P h$ & Pérdidas de núcleo, en W. \\
\hline$T f$ & $\begin{array}{l}\text { Temperatura media de las fases del } \\
\text { estator, en }{ }^{\circ} \mathrm{C} \text {. }\end{array}$ \\
\hline$T h$ & $\begin{array}{l}\text { Temperatura media del acero del } \\
\text { núcleo del estator, en }{ }^{\circ} \mathrm{C} \text {. }\end{array}$ \\
\hline Gfh & $\begin{array}{l}\text { Conductancia térmica entre el devanado } \\
\text { y el acero del estator, en } \mathrm{W} /{ }^{\circ} \mathrm{C} \text {. }\end{array}$ \\
\hline Gamb & $\begin{array}{l}\text { Conductancia térmica entre el núcleo } \\
\text { y el ambiente, en } \mathrm{W} /{ }^{\circ} \mathrm{C} \text {. }\end{array}$ \\
\hline$C f$ & $\begin{array}{l}\text { Capacitancia térmica del devanado } \\
\text { del estator, en } \mathrm{J} /{ }^{\circ} \mathrm{C} \text {. }\end{array}$ \\
\hline $\mathrm{Ch}$ & $\begin{array}{l}\text { Capacitancia térmica del núcleo del } \\
\text { estator, en } \mathrm{J} /{ }^{\circ} \mathrm{C} \text {. }\end{array}$ \\
\hline $\operatorname{Pr}$ & Pérdidas de cobre del rotor, en W. \\
\hline $\operatorname{Prc}$ & Pérdidas de núcleo del rotor, en W. \\
\hline $\operatorname{Tr}$ & $\begin{array}{l}\text { Temperatura media del devanado del } \\
\text { rotor, en }{ }^{\circ} \mathrm{C} \text {. }\end{array}$ \\
\hline $\operatorname{Trc}$ & $\begin{array}{l}\text { Temperatura media del acero del } \\
\text { núcleo del rotor, en }{ }^{\circ} \mathrm{C} \text {. }\end{array}$ \\
\hline Grc & $\begin{array}{l}\text { Conductancia térmica entre el devanado } \\
\text { y el acero del rotor, en } \mathrm{W} /{ }^{\circ} \mathrm{C} \text {. }\end{array}$ \\
\hline $\mathrm{Cr}$ & $\begin{array}{l}\text { Capacitancia térmica del devanado } \\
\text { del rotor, en } \mathrm{J} /{ }^{\circ} \mathrm{C} \text {. }\end{array}$ \\
\hline $\mathrm{Crc}$ & $\begin{array}{l}\text { Capacitancia térmica del núcleo del } \\
\text { rotor, en } \mathrm{J} /{ }^{\circ} \mathrm{C} \text {. }\end{array}$ \\
\hline
\end{tabular}


a las conductancias térmicas entre el devanado y el núcleo y entre éste y el ambiente.

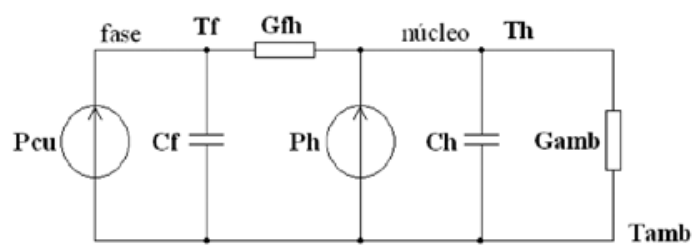

Figura 1. Circuito del modelo térmico del estator.

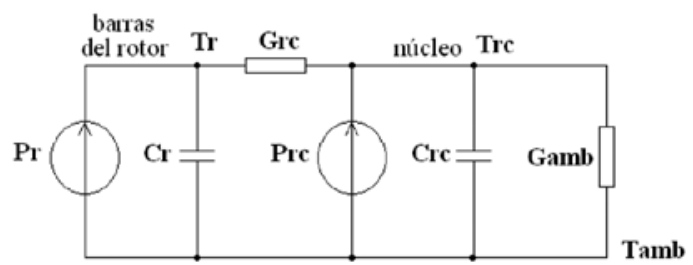

Figura 2. Circuito del modelo térmico del rotor.

Aplicando la primera ley de Kirchoff a los nodos de fase y núcleo en la Figura 1 se tiene:

$$
\begin{gathered}
-P c u+(T f-T h) \cdot G f h+C f \cdot \frac{d T f}{d t}=0 \\
-P h+(T h-T f) \cdot G f h+T h \cdot G a m b+C h \cdot \frac{d T h}{d t}=0
\end{gathered}
$$

Las ecuaciones (1) y (2) pueden reordenarse como:

$$
\frac{d T f}{d t}=\frac{[P c u-(T f-T h) \cdot G f h]}{C f}
$$

$\mathrm{y}$

$$
\frac{d T h}{d t}=\frac{[P h-(T h-T f) \cdot G f h-T h \cdot G a m b]}{C h}
$$

Considerando $T f(0)=0$ y $T h(0)=0$ (condiciones iniciales iguales a cero), o sea, que en el instante $t=0$ el motor se encuentra a la temperatura ambiente las soluciones de las ecuaciones diferenciales (3) y (4) son:

$$
\begin{gathered}
T f=\left(\frac{P c u}{G f h}+T h\right) \cdot\left(1-e^{\frac{-t \cdot G f h}{C f}}\right) \\
T h=\left(\frac{P h+G f h \cdot T f}{G a m b+G f h}+T h\right) \cdot\left(1-e^{\frac{-t \cdot(G a m b+G f h)}{C h}}\right)
\end{gathered}
$$

Como no se dispone de sensores de temperatura para medir $T h$, se recurre a simplificar el modelo. Para esto se sustituye (6) en (5), se agrupan los términos y se reduce el modelo obteniendo la expresión analítica para calcular el incremento de temperatura media del devanado con relación a la del ambiente, ver ecuación (7).

Para determinar la temperatura del devanado utilizando (7), se requiere conocer las distintas conductancias térmicas $(G)$, las capacitancias $(C)$ y las fuentes de calor $(P)$. Las pérdidas de cobre del estator y las pérdidas de núcleo son las fuentes de calor y pueden ser conocidas a partir de mediciones, utilizando un modelo eléctrico del motor para el estado de carga analizado [1314]. A continuación se propone cómo estimar las conductancias y capacitancias térmicas sin recurrir a ensayos complicados.

\section{Determinación de parámetros del circuito térmico equivalente}

Las distintas conductancias térmicas (o resistencias), y las capacitancias pueden ser aproximadamente calculadas a partir de expresiones analíticas [4]. Debido a que las conductancias y los coeficientes de transferencia de calor por convección y radiación son dependientes de varios factores geométricos, del sistema de enfriamiento y de la temperatura media del ambiente (al menos para análisis transientes), es beneficioso determinarlos mediante ensayos. Sin embargo, otra alternativa es emplear la técnica

$$
T f=\frac{\left[\frac{P c u}{G f h}+\left(\frac{P h}{G a m b+G f h}\right) \cdot\left(1-e^{\frac{-t \cdot(G a m b+G f h)}{C h}}\right)\right] \cdot\left(1-e^{\frac{-t \cdot G f h}{C f}}\right)}{1\left(\frac{G f h}{G a m b+G f h}\right) \cdot\left(1-e^{\frac{-t \cdot(G a m b+G f h)}{C h}}\right) \cdot\left(1-e^{\frac{-t \cdot G f h}{C f}}\right)}
$$


de un modelo de referencia, que está basada en minimizar un criterio de comportamiento, generalmente una función objetivo $\varepsilon$. Por lo tanto, las temperaturas experimentales y calculadas son empleadas por un algoritmo de optimización para ajustar los parámetros del modelo. El procedimiento continúa hasta que no hay mejora apreciable en el valor de la función objetivo.

En la Figura 3 se muestra un diagrama de bloques del lazo iterativo general con el criterio acompañante y la formación del modelo de referencia, mediante optimización sucesiva, donde: $\Delta T$ es el incremento de temperatura del motor en función del tiempo, $[G]$ es el vector de conductancias térmicas del modelo y $[C]$ es el vector de capacitancias térmicas.

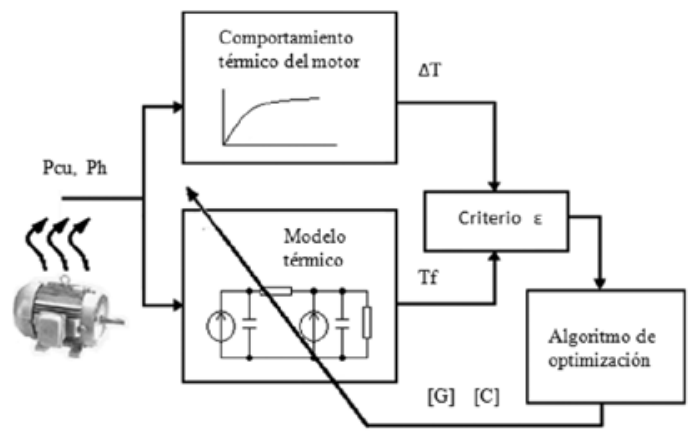

Figura 3. Diagrama de bloques del método de identificación paramétrica.

Para conformar el criterio de comportamiento es necesario obtener, con los valores experimentales, la función de incremento de temperatura $\Delta T$ en función del tiempo. Con este propósito se determina el incremento de temperatura a intervalos regulares de tiempo $(t)$, desde el momento de arranque hasta que se estabiliza la temperatura. Los valores de $\Delta T$ se calculan a partir de medir la resistencia del devanado en los intervalos deseados de $t$ y se despeja $\Delta T$ para cada caso de la expresión:

$$
R(T)=R_{a m b} \cdot\left[1+\frac{1}{273} \cdot \Delta T\right]
$$

Donde:

$R(T)$ es la resistencia del devanado medida a la temperatura T, en $\Omega$.

$R_{a m b}$ es la resistencia del devanado medida a temperatura ambiente, en $\Omega$.

$\Delta T$ es el incremento de temperatura medio del devanado con relación al ambiente, en ${ }^{\circ} \mathrm{C}$.
La medición de $R(T)$ se realiza por los procedimientos establecidos en las normas vigentes IEEE Std 1181978 y la IEEE Std 119-1974.

Como se acepta que aproximadamente el calentamiento del motor, si se considera como un cuerpo homogéneo, puede ser modelado como una ecuación exponencial, el incremento de temperatura experimental puede expresarse por:

$$
\Delta T=\Delta T_{s s} \cdot\left(1-e^{\frac{-t}{\tau}}\right)
$$

Donde:

$\Delta T_{s s}$ es el incremento de temperatura final o en condición de estado estable, en ${ }^{\circ} \mathrm{C}$.

$\tau$ es la constante de tiempo de calentamiento, en s.

En el acápite en que se valida el método, se explica cómo se determina $\Delta T_{s s}$ y $\tau$ a partir de ajuste de curva.

El criterio de comportamiento o función objetivo a optimizar es la diferencia entre la curva experimental $\Delta T$ de (9) (obtenida aplicando algún método numérico de ajuste de curvas a los datos experimentales) y la expresión del modelo térmico $T f$ dada por (7), o sea:

$$
\varepsilon=\sum_{t=0}^{n}|\Delta T-T f|
$$

El algoritmo de optimización empleado es un algoritmo genético simple (AG) implementado en Matlab. El AG es un método de optimización inspirado en la noción de evolución biológica por medio de la selección natural y ha sido utilizado por los autores en otras aplicaciones de identificación de modelos con buenos resultados [13-14]. Para el caso que se trata puede ser resumido en los siguientes pasos:

\section{a) Inicialización de la población.}

La población inicial se obtiene generando $\mu$ individuos de manera aleatoria dentro del espacio de búsqueda $\Delta X=[\Delta \text { Gfh } \Delta \text { Gamb } \Delta C h \Delta C f]^{T}$, donde los intervalos de búsqueda dependen del caso de estudio. Cada individuo de la población es 
una solución candidata representado por el vector $x^{k}=\left[G f h^{k} G a m b^{k} C h^{k} C f^{k}\right]$ para $k=1,2, \ldots, \mu$. Para esta aplicación, se tomó $\mu=100$. Por otra parte, el espacio de búsqueda queda restringido por las siguientes condiciones: $x^{k} \geq 0$ y $x(3)^{k} \geq 2 x(4)^{k}$. La primera restricción indica que todos los parámetros del modelo térmico investigado son magnitudes positivas y la segunda está relacionada con el hecho de que normalmente en las máquinas comerciales, la capacidad de almacenamiento de calor en el acero $C h$ es mayor que la capacidad de almacenamiento del devanado $C f$.

\section{b) Cruzamiento.}

El operador de cruzamiento permite mezclar información de los individuos progenitores y transmitirla a la descendencia. Existen muchos esquemas de cruzamiento disponibles en el toolbox de AG del Matlab. El operador de cruzamiento denominado heurístico (@crossoverheuristic) le adiciona una pequeña cantidad del mejor padre al peor.

Si parent1 y parent 2 son los individuos escogidos como padres y parent 1 es el que mejor valor de la función de adaptación tiene, entonces el individuo que resulta del cruzamiento se expresa matemáticamente como: child $=$ parent $2+R \cdot($ parent $1-$ parent 2$)$, donde $\mathrm{R}$ es la razón de cruzamiento, que en este caso se toma igual a 1,5 .

\section{c) Mutación.}

Las opciones de mutación especifican cómo tienen lugar pequeños cambios aleatorios en los individuos de una población. El propósito es proveer diversidad genética permitiendo que el AG explore todo el espacio de búsqueda. El operador seleccionado en este caso es@mutationadaptfeasible.

\section{d) Selección.}

La selección se lleva a cabo por la estrategia denominada uniforme estocástica (@ selectionstochunif).Este tipo de selección diseña una línea en la cual cada padre es asignado a una división de la línea de longitud proporcional a su valor escalado. El algoritmo se mueve a lo largo de la línea con pasos de igual tamaño. A cada paso el algoritmo ubica un padre de la división en la que se detiene. El primer paso es un número aleatorio uniforme menor que el tamaño del paso.

El AG cuenta con otras opciones de reproducción como el elitismo, que en este caso está presente con el operador (EliteCount). Este operador especifica el número de individuos a los que se garantiza supervivencia en la generación siguiente y que en este caso es 2. Por otro lado, la fracción de cruzamiento (CrossoverFraction) determina la fracción de la próxima generación, excluyendo a los individuos élites, que se produce por cruzamiento. Se selecciona en esta aplicación un valor de 0,8 .

\section{e) Parada.}

Los pasos del $\mathrm{b}$ al $\mathrm{d}$ son repetidos hasta que no existe mejora apreciable en el valor de la función objetivo. O sea, el algoritmo se detiene si el valor de la función objetivo no cambia durante un periodo de tiempo igual al establecido por la opción StallTimeLimit que en este caso es de $320 \mathrm{~s}$, si durante 100 generaciones este valor no tiene mejora como se ha establecido por la opción StallGenLimit, o si alcanza valores del orden de $1 \times 10^{-15}$ especificados en la opción FitnessLimit.

\section{Validación del método}

El método propuesto se valida empleando los resultados que se reportan en [1], donde se muestran las curvas de incremento de temperatura de un motor asincrónico de $3 \mathrm{~kW}, 50 \mathrm{~Hz}$, ensayado en régimen balanceado a 100 y $75 \%$ de carga. Las pérdidas de núcleo y de cobre a plena carga son $P h=170 \mathrm{~W}$ y $P c u=554,1 \mathrm{~W}$ respectivamente. Las mediciones de temperatura para estas condiciones se muestran en la Tabla 2.

Tabla 2. Incremento de temperatura medida por resistencia (de la Figura 9 de [1]).

\begin{tabular}{|c|c|c|c|c|c|c|c|c|c|}
\hline $\mathbf{t}(\mathrm{s})$ & ষ্ల & ஜ & \begin{tabular}{l}
8 \\
\hdashline \\
\end{tabular} & $\frac{8}{\stackrel{2}{d}}$ & $\frac{8}{\stackrel{2}{~}}$ & ల్ల & ஓి & $\begin{array}{l}8 \\
\stackrel{n}{7}\end{array}$ & $\frac{8}{n}$ \\
\hline $\begin{array}{c}\Delta \mathbf{T} \\
\left({ }^{\circ} \mathbf{C}\right) \\
100 \% \\
\text { carga }\end{array}$ & 28 & 46 & 55 & 62 & 66 & 68 & 70 & 72 & 72,5 \\
\hline $\begin{array}{c}\Delta \mathbf{T} \\
\left({ }^{\circ} \mathbf{C}\right) \\
75 \% \\
\text { carga }\end{array}$ & 20 & 33 & 40 & 44 & 46 & 47 & 47,1 & 47,2 & 47,2 \\
\hline
\end{tabular}


Para comprobar la generalidad del modelo que se obtiene, los parámetros del circuito térmico equivalente se determinan a partir de los datos experimentales al $100 \%$ de carga y con ellos se simula el incremento de temperatura a otros estados.

El primer paso es obtener la ecuación exponencial que describe el incremento de temperatura del devanado ajustando los datos de la Tabla 2 por la expresión (9) para $100 \%$ de carga. El ajuste se realiza empleando la opción "Curve Fitting Tool" del Matlab. Los resultados del ajuste son los siguientes:

General model:

$$
f(x)=a^{*}(1-\exp (-x / b))
$$

Coefficients (with $95 \%$ confidence bounds):

$$
\begin{aligned}
& a=72,46(70,45 ; 74,46) \\
& b=1082(961,9 ; 1203)
\end{aligned}
$$

Goodness of fit:

SSE: 26

R-square: 0,9951

Adjusted R-square: 0,9945

RMSE: 1,7

Con los valores de $\Delta T_{s s}=72,46{ }^{\circ} \mathrm{C}$ y $\tau=1082 \mathrm{~s}$, se construye la función objetivo o función de adaptación del AG dada por (10), que ofrece una medida de la calidad de la solución evaluada. El resultado de la optimización llevada a cabo por el AG se asume como el vector de parámetros del circuito térmico equivalente. Para el estudio de caso, los parámetros obtenidos por el procedimiento descrito son los siguientes:

$\mathrm{Gfh}=31 \mathrm{~W} /{ }^{\circ} \mathrm{C} ; \mathrm{Gamb}=13 \mathrm{~W} /{ }^{\circ} \mathrm{C} ; \mathrm{Ch}=33019$ $\mathrm{J} /{ }^{\circ} \mathrm{C} ; \mathrm{Cf}=6388 \mathrm{~J} /{ }^{\circ} \mathrm{C}$

En las Figuras 4 y 5 se muestran los valores de incremento de temperatura del devanado del estator medidos $(*)$ y calculados (curva de trazos discontinuos) a 100 y $75 \%$ de carga respectivamente. Como es de esperar, la mejor concordancia entre las mediciones y los valores resultantes del modelo se obtienen para el caso de plena carga, que es el que sirvió de base para la obtención de los parámetros.

El modelo térmico predice la elevación de temperatura de estado estable con una diferencia menor de $4,5{ }^{\circ} \mathrm{C}$ para el otro estado de carga diferente al que sirvió de base para la obtención del modelo. Para régimen dinámico las discrepancias son mayores, como se aprecia en las figuras.

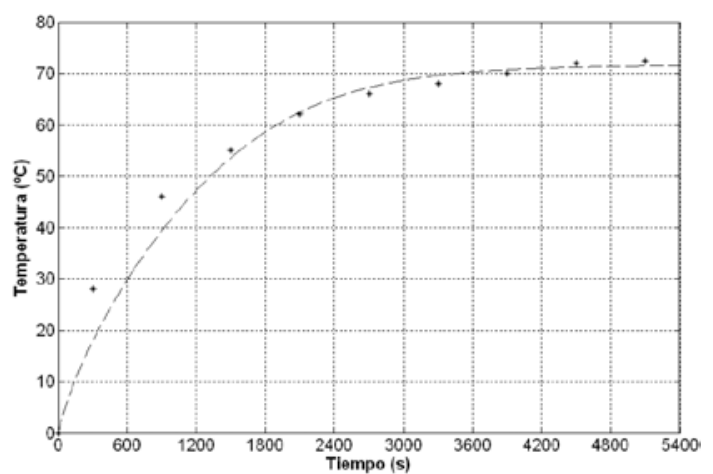

Figura 4. Curva de incremento de temperatura a $100 \%$ de carga.

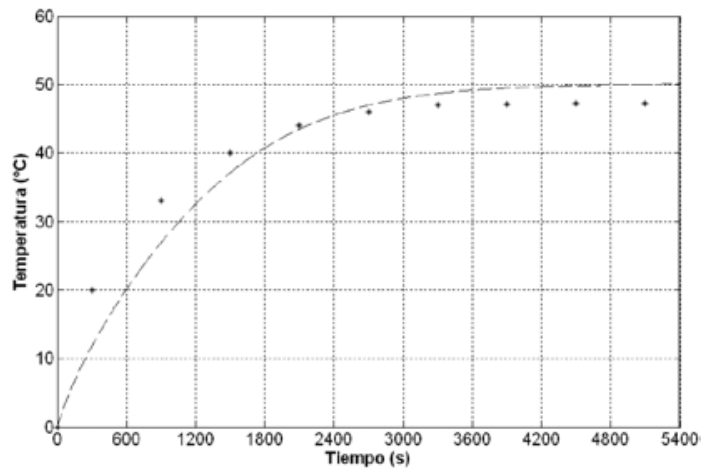

Figura 5. Curva de incremento de temperatura a $75 \%$ de carga.

Para mejorar la respuesta del modelo en régimen dinámico, se pueden ajustar directamente los datos experimentales al modelo de segundo orden de la ecuación (7) y utilizar los parámetros obtenidos anteriormente para insertarlos en la población inicial reduciendo el espacio de búsqueda al entorno de estos valores.

Aunque teóricamente los AG son capaces de encontrar óptimos globales, en problemas de optimización es importante que la población inicial contenga individuos con material genético de buena calidad. En la mayoría de las aplicaciones de AG, la población inicial se genera aleatoriamente, pero, en este trabajo, la modificación propuesta implica que la población inicial se construya heurísticamente implantando repetidamente en la misma un individuo que constituye una solución aproximada del problema; lo que garantiza que la búsqueda quede establecida en un entorno con significado físico real del modelo. 
Para el nuevo AG, los valores de $\Delta T$ en la expresión (10) no son los obtenidos de (9), sino de los valores experimentales. Los nuevos parámetros encontrados utilizando de nuevo el AG desarrollado son:

$\mathrm{Gfh}=18 \mathrm{~W} /{ }^{\circ} \mathrm{C} ; \mathrm{Gamb}=17 \mathrm{~W} /{ }^{\circ} \mathrm{C} ; \mathrm{Ch}=39896$ $\mathrm{J} /{ }^{\circ} \mathrm{C} ; \mathrm{Cf}=3421 \mathrm{~J} /{ }^{\circ} \mathrm{C}$.

Con estos parámetros la respuesta del modelo se ajusta mejor a los datos experimentales tanto en régimen dinámico como en estado estable como se aprecia en la Figura 6.

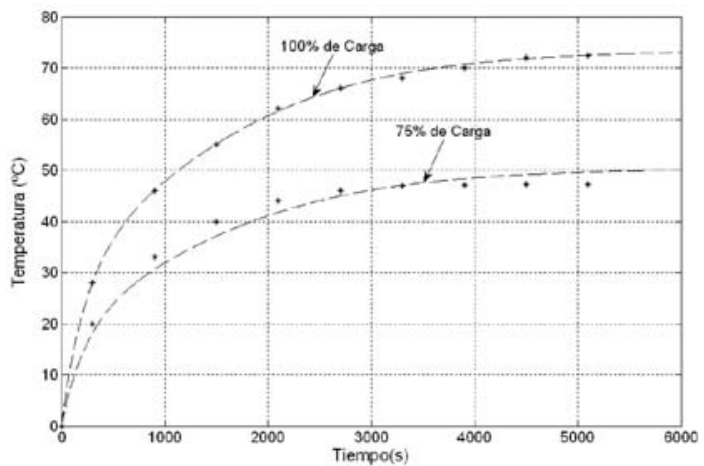

Figura 6. Curvas de incremento de temperatura para 75 y $100 \%$ de carga con los parámetros mejorados.

En la Tabla 3 se resumen los incrementos de temperatura de estado estable desde vacío hasta plena carga.

Tabla 3. Temperatura para estado estable.

\begin{tabular}{|c|c|c|c|c|}
\hline \multirow[b]{2}{*}{ Carga } & \multirow[b]{2}{*}{$\begin{array}{c}\text { Pérdidas } \\
\text { de cobre } \\
\text { del } \\
\text { estator } \\
\text { W }\end{array}$} & \multicolumn{2}{|c|}{$\begin{array}{c}\text { Incremento de } \\
\text { temperatura }\end{array}$} & \multirow[b]{2}{*}{ ט } \\
\hline & & 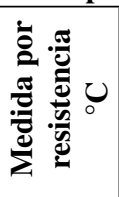 & 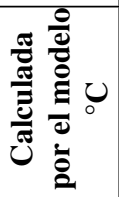 & \\
\hline Vacío & 69,6 & 19,8 & 17,95 & 1,85 \\
\hline $50 \%$ & 136 & 31,4 & 25,54 & 5,86 \\
\hline $75 \%$ & 352,3 & 47,2 & 50,28 & 3,08 \\
\hline $100 \%$ & 554,1 & 72,5 & 73,36 & 0,86 \\
\hline
\end{tabular}

De la expresión (6) se puede estimar el incremento de temperatura teórico del acero del estator. Para estado estable (6) se reduce a:

$$
T h=\frac{P h+G f h \cdot T f}{G a m b+G f h}
$$

Con esta expresión se estima que el incremento de temperatura del núcleo del estator será de $42,58{ }^{\circ} \mathrm{C}$ y de $30,71{ }^{\circ} \mathrm{C}$ para 100 y $75 \%$ de carga respectivamente.

\section{Caso de estudio}

Con el objetivo de mostrar la implementación práctica del método se realizaron mediciones de incremento de temperatura en los devanados de un motor de $1850 \mathrm{~kW}$; 6000V; 200 A; conexión Y; 3585 rpm; $\cos \varphi=0,91 ; \eta=97,1$; régimen $\mathrm{S} 1$; aislamiento clase $\mathrm{F}$, que acciona una bomba de alimentar agua a un generador de vapor (Figura 7).

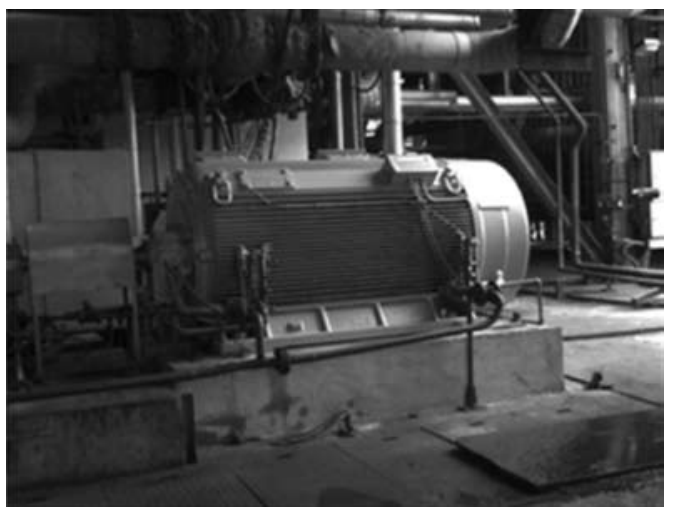

Figura 7. Motor de $1850 \mathrm{~kW}$ considerado para el estudio.

Con $\mathrm{Ph}=22,36 \mathrm{~kW}$ y $\mathrm{Pcu}=6,22 \mathrm{~kW}$ en las condiciones de carga a las cuales trabaja el motor, se ajustan los datos de incremento de temperatura obteniendo la expresión: $\Delta T=89,28 \cdot\left(1-e^{\frac{-t}{2849}}\right)$ con un coeficiente de correlación $\mathrm{R}^{2}=97,5$.

Con esta expresión de $\Delta T$ se construye la función de adaptación del AG según (10) y se obtienen los parámetros aproximados del modelo térmico propuesto. Estos valores se insertan en la población inicial de un AG para afinar la búsqueda y obtener los parámetros que se asumen como definitivos y con los cuales la respuesta del modelo es representativa, tanto del régimen dinámico como estable. 
Los parámetros definitivos, utilizando ahora en la función de adaptación (10) los valores de $\Delta T$ experimentales son:

$\mathrm{Gfh}=200 \mathrm{~W} /{ }^{\circ} \mathrm{C} ; \mathrm{Gamb}=400 \mathrm{~W} /{ }^{\circ} \mathrm{C} ; \mathrm{Ch}=3793700 \mathrm{~J} /$ ${ }^{\circ} \mathrm{C} ; \mathrm{Cf}=69300 \mathrm{~J} /{ }^{\circ} \mathrm{C}$.

En la Figura 8 se aprecia la respuesta del modelo con estos parámetros y en la Figura 9 se muestran los errores porcentuales del modelo con relación a las temperaturas experimentales.

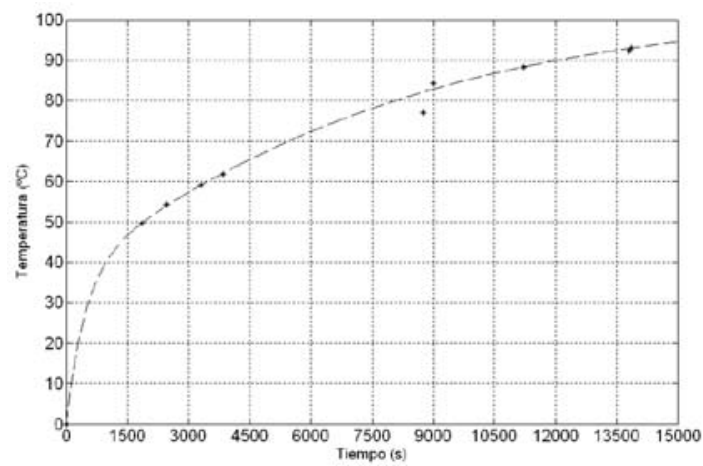

Figura 8. Curva de incremento de temperatura dada por el modelo (curva de trazos discontinuos) y los datos experimentales (*) para el motor analizado.

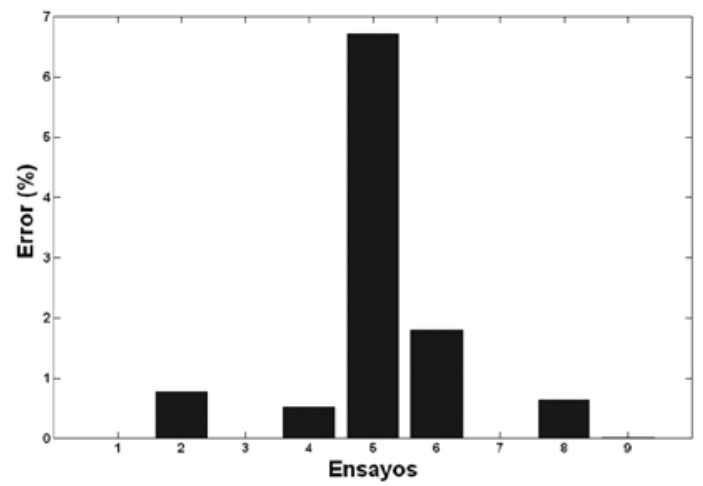

Figura 9. Errores observados en la respuesta del modelo con relación a las temperaturas experimentales.

\section{CONCLUSIONES}

En este trabajo se ha desarrollado un método para predecir el incremento de temperatura del estator del motor asincrónico, tanto para régimen dinámico como en condiciones de estabilidad térmica. Los ensayos que se proponen son sencillos de realizar en condiciones propias de la industria. Se reducen a mediciones de la resistencia del devanado del estator a intervalos regulares para determinar la curva de calentamiento a un estado de carga especificado. La menor invasividad de este procedimiento con relación a otras técnicas reportadas en la literatura hace atractivo su empleo en el análisis de calentamiento de estas máquinas.

A pesar de que no se proponen ensayos complejos para precisar los parámetros del circuito térmico equivalente, se observa una buena correlación entre los valores calculados por el modelo y los valores experimentales, tanto para el caso base reportado en [1] como para el caso de estudio mostrado.

\section{REFERENCIAS}

[1] P. Pillay and M. Manyage. "Loss of Life in Induction Machines Operating with Unbalanced Supplies". IEEE Transactions on Energy Conversion. Vol. 21, Issue 4, pp. 813-822. December, 2006.

[2] P. Gnacinski. "Windings Temperature and Loss of Life of an Induction Machine Under Voltage Unbalance Combined With Over-or Undervoltages". IEEE Transactions on Energy Conversion. Vol. 23, Issue 2, pp. 363-371. June, 2008.

[3] H. Oraee. "A Quantative Approach to Estimate the Life Expectancy of Motor Insulation Systems". IEEE Transactions on Dielectrics and Electrical Insulation. Vol. 7, Issue 6, pp. 790-796. December, 2000.

[4] A. Boglietti, A. Cavagnino and D. Staton. "Determination of Critical Parameters in Electrical Machine Thermal Models". IEEE Transactions on Industry Applications. Vol. 44, Issue 4, pp. 1150-1159. July/August, 2008.

[5] E.R. Filho and E. Avólio. "Squirrel-Cage Induction Motor Dynamics Simulation Using an Electrical and Thermal Mathematical Model Based on Manufacturer Technical Bulletins Data and on Technical Standard Statements". International Journal of Power and Energy Systems. Vol. 14, Issue 1, pp. 13-16. 1994.

[6] M.J. Durán and J. Fernández. "LumpedParameter Thermal Model for Induction Machines". IEEE Transactions on Energy 
Conversion. Vol. 19, Issue 4, pp. 791-792. December, 2004.

[7] A. Boglietti, A. Cavagnino, M. Lazzari and M. Pastorelli. "A Simplified Thermal Model for Variable-Speed Self-Cooled Industrial Induction Motor". IEEE Transactions on Industry Applications. Vol. 39, Issue 4, pp. 945-952. July/August, 2003.

[8] J.F. Moreno, F.P. Hidalgo and M.D. Martínez. "Realization of test to determine the parameters of the thermal model of an induction machine". IEE Proceedings Electric Power Applications. Vol. 148, Issue 5, pp. 393-397. September, 2001.

[9] A.L. Shenkman and M. Chertkov. "Experimental Method for Synthesis of Generalized Thermal Circuit of Polyphase Induction Motors". IEEE Transactions on Energy Conversion. Vol. 15, Issue 3, pp. 264268. September, 2000.

[10] H.A. Toliyat and G.B. Kliman. "Handbook of Electric Motors". CRC Press. Second Edition, Revised and expanded, p. 778. New York, EEUU. 2004.
[11] I. Boldea and S.A. Nasar. "The Induction Machine Handbook". CRC Press. First Edition, p. 950. 2001.

[12] O.C.N. Souto, J.C. de Oliveira and L.M. Neto. "Induction Motors Thermal Behavior and Life Expectancy Under Non-Ideal Supply Condition". Proceedings Ninth International Conference on Harmonics and Quality of Power. 2000. Vol. 3, pp. 1079-1103. August, 2002.

[13] J.R. Gómez. "Determinación de la Eficiencia de los Motores Asincrónicos con Tensiones Desbalanceadas en Condiciones de Campo". Tesis para optar al grado de doctor. Universidad Central de las Villas. Santa Clara, Cuba. 2006.

[14] J.R. Gómez, M.A. de Armas, P.R. Viego and E.C. Quispe. "Estimation of Induction Motor Efficiency In-Situ under Unbalanced Voltages Using Genetic Algorithms". XVIII International Conference on Electrical Machines, ICEM'08. Vilamoura, Portugal. 2008. 